\title{
Syzygies and diagonal resolutions for dihedral groups
}

\author{
F.E.A. Johnson
}

\begin{abstract}
Let $G$ be a finite group with integral group $\operatorname{ring} \Lambda=\mathbf{Z}[G]$. The syzygies $\Omega_{r}(\mathbf{Z})$ are the stable classes of the intermediate modules in a free $\Lambda$-resolution of the trivial module. They are of significance in the cohomology theory of $G$ via the 'co-represention theorem' $H^{r}(G, N)=\operatorname{Hom}_{\mathcal{D e r}}\left(\Omega_{r}(\mathbf{Z}), N\right)$. We describe the $\Omega_{r}(\mathbf{Z})$ explicitly for the dihedral groups $D_{4 n+2}$, so allowing the construction of free resolutions whose differentials are diagonal matrices over $\Lambda$.
\end{abstract}

Keywords: Syzygy; free resolution; diagonal resolution; dihedral group.

Mathematics Subject Classification (AMS 2010):

Primary 16E05; 20C10: Secondary 18E30

Let $\Lambda$ denote the integral group $\operatorname{ring} \Lambda=\mathbf{Z}[G]$ of a finite group $G$. We say that $\Lambda$-modules $M, M^{\prime}$ are stably equivalent (written $M \sim M^{\prime}$ ) when $M \oplus \Lambda^{a} \cong M^{\prime} \oplus \Lambda^{b}$ for some integers $a, b \geq 0$. Let

$$
\ldots \stackrel{\partial_{n+2}}{\rightarrow} F_{n+1} \stackrel{\partial_{n+1}}{\rightarrow} F_{n} \stackrel{\partial_{n}}{\rightarrow} \ldots . \stackrel{\partial_{2}}{\rightarrow} F_{1} \stackrel{\partial_{1}}{\rightarrow} F_{0} \stackrel{\partial_{0}}{\rightarrow} \mathbf{Z} \rightarrow 0
$$

be a resolution over $\Lambda$ of the trivial module $\mathbf{Z}$ in which each $F_{r}$ is a finitely generated free module. The syzygy modules $\left(J_{r}\right)_{1 \leq r}$ of $\mathcal{F}$ are the intermediate modules

$$
J_{r}=\operatorname{Im}\left(\partial_{r}\right)=\operatorname{Ker}\left(\partial_{r-1}\right) .
$$

The stable syzygy $\Omega_{r}(\mathbf{Z})$ is then defined to be the stable class $\left[J_{r}\right]$ of any such $J_{r}$. It is a standard consequence of Schanuel's Lemma (cf [6] pp. 121-122) that $\Omega_{r}(\mathbf{Z})$ is independent of the particular choice of $(\mathcal{F})$. These stable modules are of significant interest in the cohomology theory of finite groups $G$; for example, they 'co-represent' cohomology in the sense that

$$
H^{r}(G, N)=\operatorname{Hom}_{\mathcal{D e r}}\left(\Omega_{r}(\mathbf{Z}), N\right)
$$

where $\mathcal{D}$ er denotes the derived module category of $\Lambda$ (cf [5] Chap 4).

In this paper we give an explicit description of the stable syzygies $\Omega_{r}(\mathbf{Z})$ for the dihedral groups

$$
D_{4 n+2}=\left\langle x, y \mid x^{2 n+1}=1, y^{2}=1, y x y^{-1}=x^{2 n}\right\rangle .
$$

Taking $\Sigma_{x}=\sum_{r=0}^{2 n} x^{s}$, we shall show: 
(A)

$$
\Omega_{r}(\mathbf{Z}) \sim\left\{\begin{array}{ccc}
{\left[\Sigma_{x}, y-1\right] \oplus[y+1]} & r \equiv 0 & \bmod 4 \\
{[(x-1)(y-1)] \oplus[y-1]} & r \equiv 1 & \bmod 4 \\
{\left[\Sigma_{x}, y+1\right] \oplus[y+1]} & r \equiv 2 & \bmod 4 \\
{[(x-1)(y+1)] \oplus[y-1]} & r \equiv 3 & \bmod 4
\end{array}\right.
$$

where for $\alpha_{1}, \ldots, \alpha_{m} \in \Lambda,\left[\alpha_{1}, \ldots, \alpha_{m}\right]$ denotes the stable class of the right ideal

$$
\left[\alpha_{1}, \ldots, \alpha_{m}\right)=\left\{\sum_{r=1}^{m} \alpha_{i} \lambda_{i} \quad \mid \lambda_{1}, \ldots, \lambda_{m} \in \Lambda\right\} .
$$

Repetition with period four is to be expected for, as is well known ([5], [9]), the dihedral groups $D_{4 n+2}$ have cohomological period four. By contrast, periodicity is not shared by the dihedral groups of order $4 n$ and for these groups (cf [7]) the task of describing the syzygies is far more difficult and increases steadily with $r$

Taken in conjunction with periodicity the above description allows for the construction of free resolutions of an especially simple type. Thus we shall show that $D_{4 n+2}$ admits a 'diagonalised' free resolution of period four;

$$
0 \rightarrow \mathbf{Z} \stackrel{\epsilon^{*}}{\rightarrow} \Lambda \stackrel{\left(\begin{array}{c}
\partial_{3}^{+} \\
y-1
\end{array}\right)}{\longrightarrow} \Lambda \oplus \Lambda \stackrel{\left(\begin{array}{cc}
\partial_{2}^{+} & 0 \\
0^{0} & y+1
\end{array}\right)}{\longrightarrow} \Lambda \oplus \Lambda \stackrel{\left(\partial_{1}^{+}, y-1\right)}{\longrightarrow} \Lambda \stackrel{\epsilon}{\longrightarrow} \mathbf{Z} \rightarrow 0 .
$$

We may contrast this with the rather more complicated resolutions considered in [4]. The possibility of constructing diagonal resolutions for more general groups than cyclic groups was first raised in the thesis of Strouthos [8] who gave a diagonal resolution for the smallest non-abelian group, namely the dihedral group of order six.

\section{$\S 1$ : Basis calculations :}

In what follows $\Lambda$ will denote the integral group ring $\mathbf{Z}\left[D_{4 n+2}\right]$, and $\mathcal{I}$ the twosided ideal $\mathcal{I}=\mathcal{I}\left(D_{4 n+2}\right)=\operatorname{Ker}(\epsilon)$ where $\epsilon: \mathbf{Z}\left[D_{4 n+2}\right] \rightarrow \mathbf{Z}$ is the augmentation homomorphism $\epsilon(g)=1$ for $g \in D_{4 n+2}$. Throughout we work only with right modules which are also lattices over $\Lambda$; that is, $\Lambda$-modules whose underlying additive group is free abelian of finite rank. Such a right $\Lambda$-lattice $M$ determines a representation $\rho_{M}: G \rightarrow G L_{\mathbf{Z}}(M)$ by $\rho_{M}(g)(m)=m \cdot g^{-1}$.

For any finite group $G$ the operation of taking inverses induces a canonical involution on $\mathbf{Z}[G]$

$$
-: \mathbf{Z}[G] \rightarrow \mathbf{Z}[G] \quad ; \quad \overline{\sum a_{g} g}=\sum a_{g} g^{-1} .
$$

We note that $\Lambda$ contains the group ring $\mathbf{Z}\left[C_{2 n+1}\right]$ where $C_{2 n+1}$ is the cyclic group of order $2 n+1$ having generator $x$. This subring contains some distinguished elements which play a special role in our calculations. On defining

$$
\Sigma_{x}=\sum_{r=0}^{2 n} x^{r} ; \quad \theta=\sum_{r=0}^{n-1} x^{r}
$$


we note that
(1.1) $\bar{\theta}=\theta x^{n+2}$;
(1.2) $\Sigma_{x}$ is central in $\Lambda$;

Given $\alpha, \beta \in \Lambda$ we denote by $[\alpha),[\alpha, \beta)$ the right ideals

$$
\begin{array}{ccc}
{[\alpha)} & =c & \{\alpha \lambda \mid \lambda \in \Lambda\} \\
{[\alpha, \beta)} & = & \{\alpha \lambda+\beta \mu \mid \lambda, \mu \in \Lambda\} .
\end{array}
$$

We stress that any ideal in $\Lambda$ is a $\Lambda$-lattice. In what follows we shall frequently use:

Proposition 1.3 : Let $\left\{E_{\psi}\right\}_{\psi \in \Psi}$ be a $\mathbf{Z}$-basis for the free abelian group $A$ and let $B \subset A$ be an additive subgroup such that $\operatorname{rk}_{\mathbf{Z}}(B) \leq m$. Suppose also that there exists a subset $\Phi \subset \Psi$ such that $|\Phi|=m$ and $E_{\phi} \in B$ for each $\phi \in \Phi$; then

i) $\operatorname{rk}_{\mathbf{Z}}(B)=m$;

ii) $\left\{E_{\phi}\right\}_{\phi \in \Phi}$ is a $\mathbf{Z}$-basis for $B$;

iii) $A / B$ is torsion free.

We define elements $E_{r} \in \Lambda$ by



$\Lambda$ has the canonical $\mathbf{Z}=$ basis $\left\{y^{a} x^{b} \mid 0 \leq a \leq 1,0 \leq b \leq 2 n\right\}$, starting from which we proceed by elementary basis transformations to the following conclusions:

(1.4) $\left\{E_{r}\right\}_{1 \leq r \leq 4 n+2}$ is a $\mathbf{Z}$-basis for $\Lambda$.

(1.5) $\left\{E_{r}\right\}_{1 \leq r \leq 4 n+1}$ is a $\mathbf{Z}$-basis for $\mathcal{I}$.

Proposition 1.6 : $\left\{E_{r}\right\}_{1 \leq r \leq 4 n}$ is a $\mathbf{Z}$-basis for $[x-1)$.

Proof : We may regard $[x-1)$ as the induced module $[x-1)=\mathcal{I}\left(C_{2 n+1}\right) \otimes \mathbf{z}\left[C_{2 n+1} \Lambda\right.$. As $\Lambda$ is a free module of rank 2 over $\mathbf{Z}\left[C_{2 n+1}\right]$ we see that

$$
\operatorname{rk}_{\mathbf{Z}}([x-1))=2 \operatorname{rk}_{\mathbf{Z}}\left(\mathcal{I}\left(C_{2 n+1}\right)\right)=4 n .
$$

Clearly $E_{r} \in[x-1)$ for $1 \leq r \leq 2 n$ whilst

$$
E_{2 n+r}=\left(x^{2 n+1-r}-1\right) y-E_{r}=(x-1)\left(\sum_{s=0}^{2 n-r} x^{s}\right) y-E_{r} .
$$

Either way, $E_{r} \in[x-1)$ for $1 \leq r \leq 4 n$ so the result follows from (1.7) and (1.1). 
Taking $C_{2}=\left\langle y \mid y^{2}=1\right\rangle$ then a similar argument to the above using the fact that $[y-1) \cong \mathcal{I}\left(C_{2}\right) \otimes_{\mathbf{Z}\left[C_{2}\right]} \Lambda$ shows that:

(1.8) $\left\{E_{2 n+r}\right\}_{1 \leq r \leq 2 n+1}$ is a $\mathbf{Z}$-basis for $[y-1)$.

From the identities $x^{r}-1=(x-1) \sum_{s=0}^{r-1} x^{s} ; \quad y x^{r}-1=\left(x^{r}-1\right)+(y-1) x^{r}$; we observe that

$$
\mathcal{I}=[x-1)+[y-1) .
$$

As we shall see, the sum in (1.9) is far from being direct.

Proposition 1.10 $\left\{E_{2 n+r}\right\}_{1 \leq r \leq 2 n}$ is a Z-basis for $[x-1) \cap[y-1)$.

Proof: From (1.9) we obtain an exact sequence

$$
0 \rightarrow[x-1) \cap[y-1) \rightarrow[x-1) \oplus[y-1) \rightarrow \mathcal{I} \rightarrow 0
$$

from which, using (1.5), (1.6) and (1.8) we calculate that $\operatorname{rk}_{\mathbf{Z}}([x-1) \cap[y-1))=2 n$. However, from (1.6) and (1.8) we see that $E_{2 n+r} \in[x-1) \cap[y-1)$ for $1 \leq r \leq 2 n$. The result now follows from (1.3).

\section{$\S 2$ : Decomposing the augmentation ideal :}

We define elements $\pi, \rho, \widetilde{\rho} \in \Lambda$ as follows:

$$
\begin{cases}\pi= & \left(x^{n}-1\right)(y-1) \\ \rho= & (y-1)\left(x^{n+1}-x^{n}\right)=\left(x^{n}-x^{n+1}\right)(y+1) \\ \widetilde{\rho}= & (y-1)(x-1)\end{cases}
$$

Clearly $\widetilde{\rho}=\rho \cdot x^{n+1}$ and $\rho=\widetilde{\rho} \cdot x^{n}$ so that $[\rho)=[\widetilde{\rho})$. We define

$$
P=[\pi) \quad ; \quad R=[\rho]=[\widetilde{\rho}) \text {. }
$$

Evidently $\pi=(x-1)\left\{\sum_{s=0}^{n-1} x^{s}\right\}(y-1) \in[x-1)$ so that:

(2.2) $P \subset[x-1)$.

Proposition 2.3: $R=[x-1) \cap[y-1)$.

Proof : Clearly $\widetilde{\rho} \in[y-1)$ so that $R \subset[y-1)$. However, $\rho=(x-1)\left\{-x^{n}(y+1)\right\}$ so that $R \subset[x-1)$. Hence $R \subset[x-1) \cap[y-1)$. To show the opposite inclusion note that $E_{2 n+1}=\widetilde{\rho} \in R$ and $E_{2 n+r+1}=E_{2 n+1} \cdot\left\{1+x+\ldots+x^{r}\right\}$ so that $E_{2 n+r} \in R$ for $1 \leq r \leq 2 n$. Hence $[x-1) \cap[y-1) \subset R$.

Theorem 2.4 : The ideal $[x-1)$ decomposes as a direct sum 


$$
[x-1)=P+R .
$$

Proof : Put $Q=[x-1) / R$ and consider the canonical exact sequence

$$
0 \rightarrow R \hookrightarrow[x-1) \stackrel{\natural}{\rightarrow} Q \rightarrow 0 .
$$

It suffices to show that $(*)$ splits over $\Lambda$; in turn, it suffices then to show that $(* *)$ the natural map $\sqsubset$ restricts to an isomorphism $\square: P \stackrel{\simeq}{\longrightarrow} Q$.

As $R$ has the $\mathbf{Z}$-basis $\left\{E_{2 n+r}\right\}_{1 \leq r \leq 2 n}$ which extends to a basis for $\Lambda$ then $Q$ is torsion free. Furthermore, it follows from (1.6), (2.8) that:

$(* * *) \quad\left\{\left\lfloor\left(E_{r}\right)\right\}_{1 \leq r \leq 2 n}\right.$ is a $\mathbf{Z}$-basis for $Q$.

Recall that $\pi=\left(x^{n}-1\right)(y-1)$. Define $\tilde{\pi}=\pi x^{n+1}$ so that $\pi=\tilde{\pi} x^{n}$ and $[\widetilde{\pi})=[\pi]=P$. A straightforward calculation shows that

$$
\tilde{\pi}=(x-1)+(y-1)(x-1)-(y-1)\left(x^{n+1}-1\right) .
$$

Hence $\natural(\tilde{\pi})=\natural\left(E_{1}\right)$ and hence $\natural\left(\tilde{\pi} \cdot x^{r}\right)=\natural\left(E_{1} \cdot x^{r}\right)$. However

$$
E_{r}=E_{1} \cdot\left\{\sum_{s=0}^{r-1} x^{s}\right\}
$$

so that

$$
\natural\left(E_{r}\right)=\natural\left(\tilde{\pi} \cdot\left\{\sum_{s=0}^{r-1} x^{s}\right\}\right) .
$$

Thus $\downarrow: P \rightarrow Q$ is surjective and $\operatorname{rk}_{\mathbf{Z}}(P) \geq 2 n$. However $\pi \cdot y=-\pi$ so that $P=\operatorname{span}_{\mathbf{z}}\left\{\pi \cdot x^{r} \mid 0 \leq r \leq 2 n\right\}$. Moreover $\pi \cdot \Sigma_{x}=0$ so that

$$
P=\operatorname{span}_{\mathbf{z}}\left\{\pi \cdot x^{r} \mid 1 \leq r \leq 2 n\right\}
$$

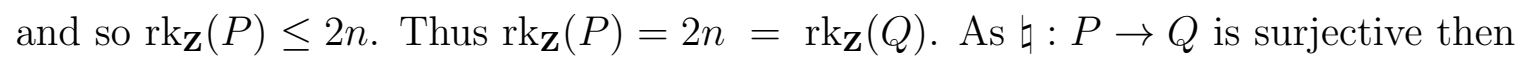
$\natural: P \rightarrow Q$ is an isomorphism as required.

We note that in the course of the above proof we established:

(2.5) $\left\{\pi \cdot x^{r}\right\}_{1 \leq r \leq 2 n}$ is a $\mathbf{Z}$-basis for $P$.

As a consequence of $(2.5)$ we have:

(2.6) $P \cap R=\{0\}$.

Corollary 2.7 : The augmentation ideal $\mathcal{I}$ decomposes as an internal direct sum

$$
\mathcal{I}=[\pi) \dot{+}[y-1) .
$$

Proof : By (1.9) we have $\mathcal{I}=[x-1)+[y-1)$ so that, by (2.4),

$$
\mathcal{I}=P+R+[y-1)
$$


However $R=[x-1) \cap[y-1) \subset[y-1)$ so that

$$
\mathcal{I}=P+[y-1)
$$

Now $P \subset[x-1)$ so that $P \cap[y-1) \subset P \cap[x-1) \cap[y-1) \subset P \cap R$. As $P \cap R=\{0\}$ then $P \cap[y-1)=\{0\}$ and $\mathcal{I}=[\pi) \dot{+}[y-1)$.

It follows from $(2.7)$ that $\mathcal{I} /[\pi)$ is torsion free. As $\Lambda / \mathcal{I} \cong \mathbf{Z}$ then :

(2.8) $\Lambda /[\pi)$ is torsion free.

\section{$\S 3$ : Characterising the modules $P$ and $R$.}

We have defined the module $P$ using a quite specific description, namely :

$$
P=\left[\left(x^{n}-1\right)(y-1)\right) \text {. }
$$

In practice, it is useful to be able to recognise when a given $\Lambda$-module is isomorphic to $P$ without being identical to the above model. Thus consider the following properties of a $\Lambda$-lattice $M$ :

$\mathcal{M}(-)$ : there exists $\widehat{\varphi}_{-} \in M$ such that $\left\{\varphi_{-} \cdot x^{r} \mid 1 \leq r \leq 2 n\right\}$ is a Z-basis for $M$ and for which $\widehat{\varphi}_{-} \cdot y=-\widehat{\varphi}_{-}$;

$\mathcal{M}(\Sigma)$ : the identity $m \cdot \Sigma_{x}=0$ holds for each $m \in M$

We recall that $P=[\pi)$ where $\pi=\left(x^{n}-1\right)(y-1)$. As $\Sigma_{x}$ is central and $\left(x^{n}-1\right) \Sigma_{x}=0$ then $P$ satisfies $\mathcal{M}(\Sigma)$. Furthermore $\pi=\left(x^{n}-1\right)(y-1)$ satisfies $\pi \cdot y=-\pi$ and, by $(2.5),\left\{\pi \cdot x^{r}\right\}_{1 \leq r \leq 2 n}$ is a $\mathbf{Z}$-basis for $P$. Thus $P$ also satisfies $\mathcal{M}(-)$. These two properties characterize $P$ up to $\Lambda$-isomorphism, as if $M$ satisfies $\mathcal{M}(-)$ and $\mathcal{M}(\Sigma)$ then $\left\{\widehat{\varphi}_{-} \cdot x^{r} \mid 1 \leq r \leq 2 n\right\}$ is a $\mathbf{Z}$-basis for $M$ and the correspondence

$$
\widehat{\varphi}_{-} \mapsto \pi \quad ; \quad \sum_{r=1}^{2 n} \widehat{\varphi}_{+} \cdot x^{r} \mapsto \sum_{r=1}^{2 n} \pi \cdot x^{r}
$$

gives an isomorphism of $\Lambda$-modules $M \stackrel{\simeq}{\longrightarrow} P$; that is:

$$
M \cong P \text { if and only if } M \text { satisfies } \mathcal{M}(-) \text { and } \mathcal{M}(\Sigma)
$$

There is a corresponding characterisation of $R$ in terms of the following property:

$\mathcal{M}(+)$ : there exists $\widehat{\varphi}_{+} \in M$ such that $\left\{\varphi_{+} \cdot x^{r} \mid 1 \leq r \leq 2 n\right\}$ is a Z-basis for $M$ and for which $\widehat{\varphi}_{+} \cdot y=\widehat{\varphi}_{+}$.

We recall that $R=[\rho)$ where $\rho=\left(x^{n}-x^{n+1}\right)(y+1)$. The module $R$ evidently satisfies $\mathcal{M}(+)$ and $\mathcal{M}(\Sigma)$. Moreover, $\rho \in R$ satisfies $\rho \cdot y=\rho$ in consequence of which $R=\operatorname{span}_{\mathbf{Z}}\left\{\rho \cdot x^{r}: 0 \leq r \leq 2 n-1\right\}$. A similar argument to the above shows: 
(3.2) $\quad M \cong R$ if and only if $M$ satisfies $\mathcal{M}(+)$ and $\mathcal{M}(\Sigma)$.

These criteria enable us to recognise non-obvious isomorphs of $P, R$; for example:

Proposition 3.3 : $\quad$ Let $a, b \in \mathbf{Z}$ be such that $a-b$ is coprime to $2 n+1$; then $\left[\left(x^{a}-x^{b}\right)(y-1)\right) \cong P$.

Proof : If $k \in \mathbf{Z}$ put $\pi(k)=\left(x^{k}-1\right)(y-1)$, so that $P=[\pi(n))$. Consider the $\Lambda$ module automorphism $\lambda: \Lambda \rightarrow \Lambda$ given by

$$
\Lambda(z)=x^{b} \cdot z
$$

Then $\lambda:[\pi(a-b)) \stackrel{\simeq}{\longrightarrow}\left[\left(x^{a}-x^{b}\right)(y-1)\right.$ is a $\Lambda$-isomorphism. As $[\pi(k))$ clearly satisfies $\mathcal{M}(\Sigma)$ it suffices to show that $\pi(k))$ satisfies $\mathcal{M}(-)$ when $k$ is coprime to $2 n+1$.

Thus suppose that $k$ is coprime to $2 n+1$ so that, in particular, $x^{k}$ generates $C_{2 n+1}$. As $n$ is also coprime to $2 n+1$ then $x^{n}$ also generates $C_{2 n+1}$. Hence there is an automorphism $\alpha: D_{4 n+2} \rightarrow D_{4 n+2}$ with the properties that $\alpha\left(x^{n}\right)=x^{k}$ and $\alpha(y)=y$. Let $\alpha_{*}: \Lambda \rightarrow \Lambda$ be the ring automorphism induced by $\alpha$. Then $\alpha_{*}(\pi)=\pi(k)$ so that $\alpha_{*}(P)=[\pi(k))$. Hence $\operatorname{rk}_{\mathbf{Z}}([\pi(k))=2 n$. However as

$$
\pi(k) \cdot y=-\pi(k)
$$

then $[\pi(k))=\operatorname{span}_{\mathbf{z}}\left\{\pi(k) \cdot x^{s} \mid 0 \leq s \leq 2 n\right\}$. However $\pi(k) \cdot \Sigma_{x}=0$ so that, as $\operatorname{rk}_{\mathbf{Z}}\left([\pi(k))=2 n\right.$ then $\left\{\pi(k) \cdot x^{s} \mid 1 \leq s \leq 2 n\right\}$ is a $\mathbf{Z}$-basis for $[\pi(k))$. Thus $[\pi(k))$ satisfies $\mathcal{M}(-)$ as required.

A similar argument yields the corresponding statement for $R$ :

Proposition 3.4: Let $a, b \in \mathbf{Z}$ be such that $a-b$ is coprime to $2 n+1$; then $\left[\left(x^{a}-x^{b}\right)(y+1)\right) \cong R$.

§4: The modules $K, L$ :

We define $K=\left[\Sigma_{x}, y-1\right)$ and $L=\left[\Sigma_{x}, y+1\right)$; we claim

Proposition 4.1 : $\operatorname{rk}_{\mathbf{Z}}(K)=2 n+2$ and $\Lambda / K$ is torsion free.

Proof : Put $K_{0}=\left\{(1-y) a(x) \mid a(x) \in \mathbf{Z}\left[C_{2 n+1}\right]\right\} \subset K$. We note that

$$
(y-1) x^{s} \cdot y=-(y-1) x^{2 n+1-s}
$$

from which it follows that $K_{0}$ is a $\Lambda$-submodule of $K$. Moreover, as

$$
\Sigma_{x} y=\sum_{s=0}^{2 n}(y-1) x^{s}+\Sigma_{x}
$$

it follows that $K$ is spanned over $\mathbf{Z}$ by $\left\{(y-1) x^{s} \mid 0 \leq s \leq 2 n\right\} \cup\left\{\Sigma_{x}\right\}$. However, starting from the canonical basis for $\Lambda$ and proceeding by elementary basis transformations, it is easy to see that $\left\{(y-1) x^{r} \mid 0 \leq r \leq 2 n\right\} \cup\left\{\Sigma_{x}\right\} \cup\left\{x^{s} \mid 1 \leq s \leq 2 n\right\}$ 
is a $\mathbf{Z}$-basis for $\Lambda$. It follows from (1.3) that $\left\{(y-1) x^{s} \mid 0 \leq s \leq 2 n\right\} \cup\left\{\Sigma_{x}\right\}$ is a $\mathbf{Z}$-basis for $K$ and $\Lambda / K$ is torsion free

For future reference we note that we have also shown:

(4.2) $\left\{(y-1) x^{s} \mid 0 \leq s \leq 2 n\right\} \cup\left\{\Sigma_{x}\right\}$ is a $\mathbf{Z}$-basis for $K$.

Proposition 4.3 $K$ is monogenic, generated by $(1-y) \theta+\Sigma_{x} y$;

Proof : It is clear that $(1-y) \theta+\Sigma_{x} y \in\left[\Sigma_{x}, y-1\right)$. However, the identity

$$
\left\{(1-y) \theta+\Sigma_{x} y\right\} \cdot x^{n+1}(1-y)=(y-1)
$$

shows that $(y-1) \in\left[(1-y) \theta+\Sigma_{x} y\right)$. Thus $(y-1) \theta \in\left[(1-y) \theta+\Sigma_{x} y\right)$. Hence $\Sigma_{x} y \in\left[(1-y) \theta+\Sigma_{x} y\right)$ and so $\Sigma_{x}=\left\{\Sigma_{x} y\right\} \cdot y \in\left[(1-y) \theta+\Sigma_{x} y\right)$.

Now put $L_{0}=\left\{(y+1) a(x) \mid a(x) \in \mathbf{Z}\left[C_{2 n+1}\right]\right\} \subset L$; similarly to (4.1) we have:

Proposition 4.4: $\operatorname{rk}_{\mathbf{Z}}(L)=2 n+2$ and $\Lambda / L$ is torsion free.

Furthermore:

(4.5) $\left\{(y+1) x^{s} \mid 0 \leq s \leq 2 n\right\} \cup\left\{\Sigma_{x}\right\}$ is a $\mathbf{Z}$-basis for $L$.

Noting that $\left\{(1+y) \theta-\Sigma_{x} y\right\} \cdot x^{n+1}(y+1)=-(y+1)$ an analogous argument to (4.3) then shows that:

Proposition 4.6 $L$ is monogenic, generated by $(1+y) \theta-\Sigma_{x} y$.

\section{$\S 5$ : Two diagonal resolutions :}

Define elements in $\Lambda$ as follows

$$
\begin{aligned}
& \partial_{0}^{+}=(1-y) \theta+\Sigma_{x} \cdot y ; \\
& \partial_{1}^{+}=\left(x^{n+1}-x\right)(y-1) ; \\
& \partial_{2}^{+}=(1+y) \theta-\Sigma_{x} \cdot y ; \\
& \partial_{3}^{+}=\left(x^{n+1}-x\right)(y+1)
\end{aligned}
$$

and put $\partial_{4}^{+}=\partial_{0}^{+}$; we have a sequence repeating with period four infinitely in both directions:

$$
\ldots \stackrel{\partial_{1}^{+}}{\rightarrow} \Lambda \stackrel{\partial_{0}^{+}}{\rightarrow} \Lambda \stackrel{\partial_{3}^{+}}{\rightarrow} \Lambda \stackrel{\partial_{2}^{+}}{\rightarrow} \Lambda \stackrel{\partial_{1}^{+}}{\rightarrow} \Lambda \stackrel{\partial_{0}^{+}}{\rightarrow} \Lambda \stackrel{\partial_{3}^{+}}{\rightarrow} \ldots
$$

We shall show that $\left(\mathcal{S}^{+}\right)$is exact. To do this first observe that: 
Proposition 5.1: $\partial_{0}^{+} \partial_{1}^{+}=0$.

Proof : From the above definitions we see that $\partial_{0}^{+} \partial_{1}^{+}=A+B$ where

$$
A=(1-y) \theta\left(x^{n+1}-x\right)(y-1) \quad ; \quad B=\Sigma_{x} y\left(x^{n+1}-x\right)(y-1) .
$$

As $\Sigma_{x}$ is central in $\Lambda$ and $\Sigma_{x}\left(x^{n+1}-x\right)=0$ it follows that $B=0$.

To show that $A=0$ we first note that

$$
\begin{aligned}
A & =(1-y) y\left\{\overline{\theta x^{n+1}}-\overline{\theta x}\right\}+(y-1)\left\{\theta x^{n+1}-\theta x\right\} \\
& =(y-1)\left\{\left(\overline{\theta x^{n+1}}-\theta x\right)+\left(\theta x^{n+1}-\overline{\theta x}\right)\right\} .
\end{aligned}
$$

However, by (1.1), $\bar{\theta}=\theta x^{n+2}$ so that $\overline{\theta x^{n+1}}=\theta x^{n+2} x^{n}=\theta x$ and likewise $\theta x^{n+1}=\overline{\theta x}$. As required we have $A=0$.

A similar proof shows that

$$
\partial_{2}^{+} \partial_{3}^{+}=0 \text {. }
$$

From the fact that $y^{2}=1$ and that $\Sigma_{x}$ is central in $\Lambda$ it follows that:

$$
\begin{aligned}
& \partial_{1}^{+} \partial_{2}^{+}=0 \\
& \partial_{3}^{+} \partial_{0}^{+}=0
\end{aligned}
$$

Proposition 5.5: $\quad \Lambda /\left[\partial_{r}^{+}\right)$is torsion free for each $r$.

Proof : Let $\tau: \Lambda \rightarrow \Lambda$ be the $\Lambda$-isomorphism $\tau(\lambda)=x \lambda$. Then $\tau$ induces an isomorphism $\Lambda /[\pi) \stackrel{\simeq}{\longrightarrow} \Lambda /\left[\tau(\pi)\right.$ ). However $\tau(\pi)=\partial_{1}^{+}$and $\Lambda /[\pi)$ is torsion free, by (2.8). Thus $\Lambda /\left[\partial_{1}^{+}\right)$is torsion free.

To show that $\Lambda /\left[\partial_{3}^{+}\right)$is torsion free, put $v=(x-1)(y+1)$. Then $\tau^{n}(v)=-\rho$ so that $\Lambda /[v)$ is torsion free by (1.3), (1.10) and (2.3). Observing that $n$ is coprime to $2 n+1$, let $\alpha: D_{4 n+2} \rightarrow D_{4 n+2}$ be the automorphism $\alpha(x)=x^{n} ; \alpha(y)=y$ and let $\alpha_{*}: \Lambda \rightarrow \Lambda$ be the ring automorphism induced from $\alpha$. Then $\tau \circ \alpha_{*}(v)=\partial_{3}^{+}$so that $\Lambda /\left[\partial_{3}^{+}\right)$is torsion free.

For the remaining two cases, observe that $\left[\partial_{0}^{+}\right)=K$ and $\left[\partial_{2}^{+}\right)=L$. However, $\Lambda / K$ is torsion free by (4.1) and $\Lambda / L$ is torsion free by (4.4).

Proposition $5.6: \operatorname{rk}_{\mathbf{Z}}\left[\operatorname{Ker}\left(\partial_{r}^{+}\right)\right]=\operatorname{rk}_{\mathbf{Z}}\left[\operatorname{Im}\left(\partial_{r+1}^{+}\right)\right]$for $0 \leq r \leq 3$.

Proof : Observe that

$$
\operatorname{rk}_{\mathbf{Z}}\left[\operatorname{Im}\left(\partial_{r}^{+}\right)\right]=\left\{\begin{array}{cc}
2 n+2 & r \text { even } \\
2 n & r \text { odd }
\end{array}\right.
$$


However $\operatorname{rk}_{\mathbf{Z}}\left(\operatorname{Ker}\left(\partial_{r}^{+}\right)=4 n+2-\operatorname{rk}_{\mathbf{Z}}\left(\operatorname{Im}\left(\partial_{r}^{+}\right)\right.\right.$; on applying this to $\left(^{*}\right)$ we see that

$$
\operatorname{rk}_{\mathbf{Z}}\left[\operatorname{Ker}\left(\partial_{r}^{+}\right)\right]=\left\{\begin{array}{cc}
2 n & r \text { even } \\
2 n+2 & r \text { odd }
\end{array}\right.
$$

On re-expressing $(*)$ in the following form

$(* * *)$

$$
\mathrm{rk}_{\mathbf{Z}}\left[\operatorname{Im}\left(\partial_{r+1}^{+}\right)\right]=\left\{\begin{array}{cc}
2 n & r \text { even } \\
2 n+2 & r \text { odd }
\end{array}\right.
$$

the result follows immediately.

Theorem 5.7: The sequence $\left(\mathcal{S}^{+}\right)$is exact.

Proof : $\quad$ From (5.1) - (5.4) we see that $\operatorname{Im}\left(\partial_{r+1}^{+}\right) \subset \operatorname{Ker}\left(\partial_{r}^{+}\right)$for $0 \leq r \leq 4$. From (5.6) it follows that each $\operatorname{Ker}\left(\partial_{r}^{+}\right) / \operatorname{Im}\left(\partial_{r+1}^{+}\right)$is finite. However, by (5.1) each $\operatorname{Ker}\left(\partial_{r}^{+}\right) / \operatorname{Im}\left(\partial_{r+1}^{+}\right)$is also torsion free and so $\operatorname{Ker}\left(\partial_{r}^{+}\right)=\operatorname{Im}\left(\partial_{r+1}^{+}\right)$

In addition to $\left(\mathcal{S}^{+}\right)$we have another long exact sequence $\left(\mathcal{S}^{-}\right)$of period two;

$$
\cdots \stackrel{y+1}{\rightarrow} \Lambda \stackrel{y-1}{\rightarrow} \Lambda \stackrel{y+1}{\rightarrow} \Lambda \stackrel{y-1}{\rightarrow} \Lambda \stackrel{y+1}{\rightarrow} \Lambda \stackrel{y-1}{\rightarrow} \Lambda \stackrel{y+1}{\rightarrow} \ldots
$$

From $\left(\mathcal{S}^{+}\right)$and $\left(\mathcal{S}^{-}\right)$we may form another exact sequence, again repeating with period four infinitely in both directions:

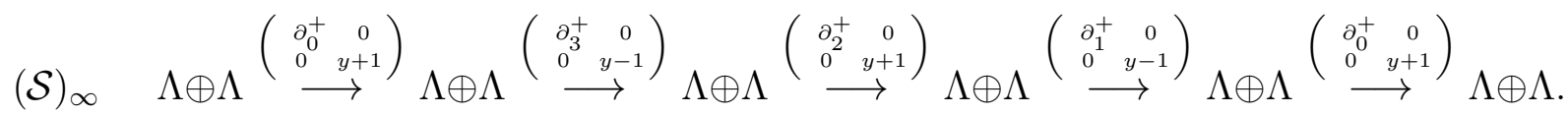

The sequence $(\mathcal{S})$ is a complete resolution of $D_{4 n+2}$ in the sense of Tate [1]. We proceed to modify $(\mathcal{S})$ in a number of ways. Taking $\epsilon: \Lambda \rightarrow \mathbf{Z}$ to be the augmentation map and defining

$$
\begin{aligned}
\partial_{1}: \Lambda \oplus \Lambda \rightarrow \Lambda & ; & \partial_{1}=\left(\partial_{1}^{+}, y-1\right) \\
\partial_{2}: \Lambda \oplus \Lambda \rightarrow \Lambda \oplus \Lambda & ; & \partial_{2}=\left(\begin{array}{cc}
\partial_{2}^{+} & 0 \\
0 & y+1
\end{array}\right)
\end{aligned}
$$

we have the following sequence

$(\mathcal{S}) \quad . \rightarrow \Lambda \oplus \Lambda \stackrel{\left(\begin{array}{cc}\partial_{1}^{+} & 0 \\ 0 & y+1\end{array}\right)}{\longrightarrow} \Lambda \oplus \Lambda \stackrel{\left(\begin{array}{cc}\partial_{0}^{+} & 0 \\ 0 & y+1\end{array}\right)}{\longrightarrow} \Lambda \oplus \Lambda \stackrel{\left(\begin{array}{cc}\partial_{3}^{+} & 0 \\ 0 & y-1\end{array}\right)}{\longrightarrow} \Lambda \oplus \Lambda \stackrel{\partial_{2}}{\rightarrow} \Lambda \oplus \Lambda \stackrel{\partial_{1}}{\rightarrow} \Lambda \stackrel{\epsilon}{\rightarrow} \mathbf{Z} \rightarrow 0$

which continues thereafter infinitely to the left with the same differentials as $(\mathcal{S})_{\infty}$. Noting that $\operatorname{Ker}(\epsilon)=\mathcal{I}$ and that, by (2.7),

$$
\mathcal{I}=[\pi) \dot{+}[y-1)=\left[\partial_{1}^{+}\right) \dot{+} \operatorname{Im}(y-1)=\operatorname{Im}\left(\partial_{1}\right)
$$


we see that:

(5.8) $\operatorname{Ker}(\epsilon)=\operatorname{Im}\left(\partial_{1}\right)$.

To proceed we note the following identity.

(5.9) $\left[(1-y) \theta+\Sigma_{x} y\right](y-1)=(y-1)$.

Proposition 5.10: $\operatorname{Ker}\left(\partial_{1}\right)=\operatorname{Im}\left(\partial_{2}\right)$.

Proof : It is straightforward to check that $\partial_{1} \partial_{2}=0$ so it suffices to show that $\operatorname{Ker}\left(\partial_{1}\right) \subset \operatorname{Im}\left(\partial_{2}\right)$. Thus suppose that

$$
\partial_{1}\left(\begin{array}{l}
a \\
b
\end{array}\right)=0
$$

Then $\partial_{1}^{+}(a)=-(y-1) b$. However, $\partial_{0}^{+} \partial_{1}^{+}=0$ so that, by the identity of (5.9),

$$
\partial_{0}^{+}(y-1) b=\left\{(1-y) \theta+\Sigma_{x} y\right\}(y-1) b=(y-1) b=0
$$

Thus $b=(y+1) d$ for some $d \in \Lambda$ and $\partial_{1}^{+}(a)=-(y-1)(y+1) d=0$. Hence $a=\partial_{2}^{+}(c)$ for some $c \in \Lambda$ and

$$
\left(\begin{array}{l}
a \\
b
\end{array}\right)=\left(\begin{array}{cc}
\partial_{2}^{+} & 0 \\
0 & y+1
\end{array}\right)\left(\begin{array}{l}
c \\
d
\end{array}\right)=\partial_{2}\left(\begin{array}{l}
c \\
d
\end{array}\right)
$$

From the foregoing we see that:

(5.11) The sequence $(\mathcal{S})$ is exact.

The sequence $(\mathcal{S})$ is a free diagonal resolution of $D_{4 n+2}$ of period four. There is, however, an even simpler free resolution to be obtained. Thus if we now define

$$
\begin{array}{ccc}
\partial_{3}: \Lambda \rightarrow \Lambda \oplus \Lambda & ; & \partial_{3}=\left(\begin{array}{c}
\partial_{3}^{+} \\
y-1
\end{array}\right) \\
\epsilon^{*}: \mathbf{Z} \rightarrow \Lambda & ; & \epsilon^{*}(1)=\Sigma_{x}(1+y)
\end{array}
$$

we obtain the following finite sequence

$(\mathcal{D})_{\text {fin }}$

$$
0 \rightarrow \mathbf{Z} \stackrel{\epsilon^{*}}{\longrightarrow} \Lambda \stackrel{\partial_{3}}{\longrightarrow} \Lambda \oplus \Lambda \stackrel{\partial_{2}}{\longrightarrow} \Lambda \oplus \Lambda \stackrel{\partial_{1}}{\longrightarrow} \Lambda \stackrel{\epsilon}{\longrightarrow} \mathbf{Z} \rightarrow 0
$$

From the definition of $\partial_{3}$ and the exactness of $(\mathcal{S})$ it follows immediately that 


$$
\operatorname{Ker}\left(\partial_{2}\right)=\operatorname{Im}\left(\partial_{3}\right) .
$$

Proposition 5.13: $\operatorname{Ker}\left(\partial_{3}\right)=\operatorname{Im}\left(\epsilon^{*}\right)$.

Proof : It is straightforward to see that $\operatorname{Im}\left(\epsilon^{*}\right) \subset \operatorname{Ker}\left(\partial_{3}\right)$. To establish the reverse inclusion, suppose $e \in \Lambda$ satisfies $\partial_{3}(e)=\left(\begin{array}{l}0 \\ 0\end{array}\right)$; then

$$
\left(x^{n+1}-x^{n}\right)(y+1) e=(y-1) e=0 .
$$

In particular, $e=(y+1) f$ and so $\left(x^{n+1}-x^{n}\right)(y+1)(y+1) f=0$; that is $2 x^{n}(x-1)(y+1) f=0$ or equivalently

$$
(x-1)(y+1) f=0 .
$$

Write $f=g(x)+h(x) y$ where $g(x), h(x) \in \mathbf{Z}\left[C_{2 n+1}\right]$ so that

$$
e=(1+y) f=\alpha(x)(1+y)
$$

where $\alpha(x)=g(x)+\overline{h(x)}$. As $(x-1) e=0$ then $(x-1) \alpha(x)=0$ so that $\alpha(x)=\lambda \Sigma_{x}$ and so $e=\lambda \Sigma_{x}(1+y)=\epsilon^{*}(\lambda)$. Thus $\operatorname{Ker}\left(\partial_{3}\right) \subset \operatorname{Im}\left(\epsilon^{*}\right)$.

In consequence of the foregoing we obtain the following, which is statement (B) of the Introduction:

(5.14) The sequence $(\mathcal{D})_{\text {fin }}$ is exact.

Observing that $\epsilon \epsilon^{*}=\Sigma_{x}(1+y)$ we may repeat $(\mathcal{D})_{\text {fin }}$ infinitely to the left to obtain another free resolution of $D_{4 n+2}$ with period four thus:

$$
\ldots \stackrel{\partial_{2}}{\longrightarrow} \Lambda \oplus \Lambda \stackrel{\partial_{1}}{\longrightarrow} \Lambda \stackrel{\Sigma_{x}(1+y)}{\longrightarrow} \Lambda \stackrel{\partial_{3}}{\longrightarrow} \Lambda \oplus \Lambda \stackrel{\partial_{2}}{\longrightarrow} \Lambda \oplus \Lambda \stackrel{\partial_{1}}{\longrightarrow} \Lambda \stackrel{\epsilon}{\longrightarrow} \mathbf{Z} \rightarrow 0 .
$$

$\S 6$ : The syzygies $\Omega_{r}^{D_{4 n+2}}(\mathbf{Z})$ :

Let $\Lambda$ denote the integral group $\operatorname{ring} \Lambda=\mathbf{Z}[G]$ of a finite group $G$. If $M$ is a $\Lambda$-module and

$$
\ldots \stackrel{\partial_{n+2}}{\longrightarrow} F_{n+1} \stackrel{\partial_{n+1}}{\longrightarrow} F_{n} \stackrel{\partial_{n}}{\longrightarrow} \ldots \ldots \stackrel{\partial_{2}}{\rightarrow} F_{1} \stackrel{\partial_{1}}{\longrightarrow} F_{0} \stackrel{\partial_{0}}{\rightarrow} M \rightarrow 0
$$

is a free resolution of $M$ of finite type over $\Lambda$ the syzygy modules $\left(J_{r}\right)_{1 \leq r}$ of $\mathcal{F}$ are the intermediate modules $J_{r}=\operatorname{Im}\left(\partial_{r}\right)=\operatorname{Ker}\left(\partial_{r-1}\right)$. The stable class $\left[\operatorname{Im}\left(\partial_{r}\right]\right.$ is independent of $(\mathcal{F})$ and is written

$$
\Omega_{r}^{G}(M)=\left[\operatorname{Im}\left(\partial_{r}\right] .\right.
$$


From the resolution $(\mathcal{D})_{\infty}$ we can read off the syzygies $\Omega_{r}(\mathbf{Z})\left(=\Omega_{r}^{D_{4 n+2}}(\mathbf{Z})\right)$ directly:

$$
\Omega_{r}(\mathbf{Z}) \sim\left\{\begin{array}{ccc}
{[\mathbf{Z}]} & r \equiv 0 & \bmod 4 \\
{\left[\left(x^{n+1}-x\right)(y-1)\right] \oplus[y-1]} & r \equiv 1 & \bmod 4 \\
{\left[(1+y) \theta-\Sigma_{x} y\right] \oplus[y+1]} & r \equiv 2 & \bmod 4 \\
{\left[\left(x^{n+1}-x\right)(y+1)\right] \oplus[y-1]} & r \equiv 3 & \bmod 4 .
\end{array}\right.
$$

This description can be simplified; as $n$ is coprime to $2 n+1$ then by (3.3) and (3.4)

$$
\left[\left(x^{n+1}-x\right)(y-1)\right) \cong P \quad ; \quad\left[\left(x^{n+1}-x\right)(y+1)\right) \cong R
$$

whilst from (4.3) and (4.6) we have $\left[(1+y) \theta-\Sigma_{x} y\right) \cong L$. Thus

$$
\Omega_{r}(\mathbf{Z}) \sim\left\{\begin{array}{ccc}
{[\mathbf{Z}]} & r \equiv 0 & \bmod 4 \\
{[P] \oplus[y-1]} & r \equiv 1 & \bmod 4 \\
{[L] \oplus[y+1]} & r \equiv 2 & \bmod 4 \\
{[R] \oplus[y-1]} & r \equiv 3 & \bmod 4 .
\end{array}\right.
$$

Reading off the syzygies from the resolution $(\mathcal{S})$ gives a slightly different expression for $\Omega_{4}(\mathbf{Z})$; recalling from $(4.3)$ that $\left[(1-y) \theta+\Sigma_{x} y\right) \cong K$, then

$$
\Omega_{4}(\mathbf{Z}) \sim[K] \oplus[y+1] .
$$

Comparing the expressions for $\Omega_{4}(\mathbf{Z})$ in $(6.1)$ and (6.3) we find that :

$$
[\mathbf{Z}]=[K] \oplus[y+1] .
$$

Together with (6.4), the isomorphisms $\left[(1-y) \theta+\Sigma_{x} y\right) \cong K,[(x-1)(y-1)) \cong P$, $\left[(1+y) \theta-\Sigma_{x} y\right) \cong L,[(x-1)(y+1)) \cong R$ show that $(6.2)$ is equivalent to the statement (A) of the Introduction.

The decomposition (6.4) illustrates a somewhat paradoxical aspect of the theory of stable modules, namely that whilst a module (in this case the trivial module $\mathbf{Z}$ ) may be indecomposable, its stable class may decompose non-trivially. This phenomenon seems first to have been pointed out, though without an explicit example, in the paper of Gruenberg and Roggenkamp ([3] Proposition 1). They attribute the original observation to E.C. Dade ([3] p. 153). 


\section{$\S 7$ : Relations between the modules :}

If $M, N$ are $\Lambda$-lattices the tensor product $M \otimes N$ is defined by imposing the group action $(m \otimes n) \cdot g=m \cdot g \otimes n \cdot g$ on the abelian group $M \otimes \mathbf{z} N$. Extending this in an obvious way to stable modules it is well known and straightforward to show that

$$
\Omega_{k}(\mathbf{Z}) \otimes \Omega_{l}(\mathbf{Z})=\Omega_{k+l}(\mathbf{Z}) .
$$

This suggests corresponding relations between the modules $K, P, L, R$. For example, the relation $\Omega_{1}(\mathbf{Z}) \otimes \Omega_{1}(\mathbf{Z})=\Omega_{2}(\mathbf{Z})$ suggests a stable equivalence $P \otimes P \sim L$. This is indeed the case. More precisely, the author's student John Evans has shown that (cf [2]), under tensor product, the relations amongst the modules $K, P, L, R$ are given by the following table.

\begin{tabular}{|c|l|l|l|l|}
\hline \hline$\otimes$ & \multicolumn{1}{c|}{$K$} & \multicolumn{1}{c|}{$P$} & \multicolumn{1}{c|}{$L$} & \multicolumn{1}{c|}{$R$} \\
\hline \hline$K$ & $K \oplus \Lambda^{n+1}$ & $P \oplus \Lambda^{n}$ & $L \oplus \Lambda^{n+1}$ & $R \oplus \Lambda^{n}$ \\
\hline$P$ & $P \oplus \Lambda^{n}$ & $L \oplus \Lambda^{n-1}$ & $R \oplus \Lambda^{n}$ & $K \oplus \Lambda^{n-1}$ \\
\hline$L$ & $L \oplus \Lambda^{n+1}$ & $R \oplus \Lambda^{n}$ & $K \oplus \Lambda^{n+1}$ & $P \oplus \Lambda^{n}$ \\
\hline$R$ & $R \oplus \Lambda^{n}$ & $K \oplus \Lambda^{n-1}$ & $P \oplus \Lambda^{n}$ & $L \oplus \Lambda^{n-1}$ \\
\hline
\end{tabular}

Thus under the operation of tensor product one may view the stable modules $[K],[P],[L],[R]$ as a cyclic group of order 4 generated either by $[P]$ or $[R]$, with $[K]$ as identity.

There are corresponding duality statements. Over an arbitrary finite group one has $\Omega_{r}(\mathbf{Z})^{*}=\Omega_{-r}(\mathbf{Z})$. However in the special case $G=D_{4 n+2}$ the syzygies have period four, $\Omega_{r}(\mathbf{Z})=\Omega_{r+4}(\mathbf{Z})$ so that

$$
\Omega_{r}^{*}(\mathbf{Z})=\Omega_{4-r}(\mathbf{Z}) .
$$

In fact the corresponding relations already hold at the level of modules, namely

(7.4) $\quad K^{*} \cong K$

(7.5) $\quad L^{*} \cong L$;

(7.6) $\quad P^{*} \cong R$;

(7.7) $\quad R^{*} \cong P$.

One should perhaps stress that no two of $K, P, L, R$ are isomorphic. In fact, given that $D_{4 n+2}$ has cohomological period four, no two of $K, P, L, R$ are stably isomorphic.

\section{F.E.A. Johnson}

Department of Mathematics

University College London

Gower Street, London WC1E 6BT, U.K.

e-mail address : feaj@math.ucl.ac.uk 


\section{REFERENCES}

[1]: M.F. Atiyah and C.T.C. Wall; Cohomology of groups; in 'Algebraic Number Theory' (edited by J.W.S. Cassels and A Fröhlich) Academic Press, 1967.

[2]: J.D.P. Evans; Ph.D Thesis. University College London (in preparation).

[3]: K.W. Gruenberg and K.W. Roggenkamp; Decomposition of the augmentation ideal and of the relation modules of a finite group. Proc. Lond. Math. Soc. 31 (1975) 149-166.

[4] : F.E.A. Johnson; Explicit homotopy equivalences in dimension two: Math. Proc. Camb. Phil. Soc. 133 (2002) 411-430.

[5]: F.E.A. Johnson; Stable modules and the D(2)-problem: LMS Lecture Notes In Mathematics, vol. 301. CUP 2003.

[6] : F.E.A. Johnson; Syzygies and homotopy theory: Springer-Verlag. 2011.

[7] : W.H. Mannan and S. O'Shea; Minimal algebraic $\pi_{2}$ over $D_{4 n}$ : Algebraic Geometry and Topology 13 (2013) 3287-3304.

[8] : I. Strouthos; Stably free modules over group rings: PhD Thesis, University College London, 2010.

[9] : R.G. Swan; Periodic resolutions for finite groups: Ann. of Math. 72 (1960) 267-291. 